\title{
Retrospectiva das dermatofitoses em cães e gatos atendidos no Hospital Veterinário da Universidade Federal de Mato Grosso, nos anos de 2006 a 2008
}

\author{
A retrospective of dermatophytosis in dogs and cats Veterinary Hospital at the Universidade Federal \\ de Mato Grosso, in the years 2006 to 2008
}

\author{
Rita de Cássia da Silva Machado Neves ${ }^{\text {"* }}$ Felipe Augusto Constantino Seabra da Cruz ${ }^{\text {II }}$ \\ Samara Rosolem Lima ${ }^{\mathrm{III}}$ Mariana Medeiros Torres ${ }^{\mathrm{I}}$ Valéria Dutra $^{\mathrm{III}}$ Valéria Régia Franco Sousa ${ }^{\mathrm{III}}$
}

\section{RESUMO}

$O$ objetivo deste estudo foi caracterizar a população canina e felina, atendida em um período de 36 meses, no Hospital Veterinário da Universidade Federal de Mato Grosso - HOVET/UFMT, acometidos por dermatofitose. Esta dermatopatia, que atinge os animais domésticos, é uma infecção fúngica que envolve camadas superficiais da pele, pêlo e unhas. Foram atendidos 279 casos de dermatofitose, 96,8\% na espécie canina e 3,2\% na espécie felina, totalizando $7,1 \%$ do total de 3096 casos consultados nestes três anos. O agente etiológico isolado preponderante foi o Microsporum canis. Os cães de raça definida foram os mais acometidos, mormente os American Pit Bull Terrier (21,7\%). Tanto os felinos sem ou com definição racial apresentaram dermatofitose, não sendo possível uma análise estatística fidedigna. Observou-se que a maioria dos animais infectados apresentava de 1 a 3 anos de idade. As lesões mais observadas foram: alopecia, crostas e caspas e estavam localizadas nas regiões da cabeça, de tronco, e de membros. Não foi observada distribuição sazonal.

Palavras-chave: dermatopatia, dermatófito, caninos e felinos.

\section{ABSTRACT}

The aim of this study was to characterize the canine and feline population with dermatophytosis, taken to the Veterinary Hospital of Universidade Federal do Mato GrossoHOVET/UFMT, during a period of 36 months. This skin disease that affects domestic animals is a fungal infection involving the superficial layers of the skin, hair and nails. 279 cases of dermatophytosis were treated, $96.8 \%$ in dogs and $3.2 \%$ in cats, with a total of $7.1 \%$ of the number of 3096 cases assisted in these three years. The predominant etiologic agent was

\begin{abstract}
Microsporum canis. The pure breddogs were most affected, especially the American Pit Bull Terrier (21.7\%). The cats with and without racial definition were dermatophytosis, cannot be a reliable statistical analysis. It was observed that the majority of animals infected were 1-3 years old. The lesions observed were: alopecia, dandruff and crusts and were located in the cephalic region, trunk, and limbs. There was no seasonal distribution was observed.
\end{abstract}

Key words: skin disease, dermatophytes, canine and felines.

\section{INTRODUÇÃO}

As dermatofitoses são um grupo de micoses cutâneas produzidas por fungos imperfeitos, Microscoporum spp. e Trichophyton spp., que penetram no extrato córneo invadindo os pêlos, as unhas e a camada córnea da pele(CAVALCANTI et al., 2003). Embora aproximadamente 30 espécies causem infecções de pele em mamíferos e aves, poucas espécies são rotineiramente isoladas. Microsporum canis é o dermatófito zoofílico provavelmente melhor adaptado ao gato, mas é a causa mais comum da dermatofitose canina. Outros dermatófitos também são isolados de cães como Microsporum gypseum e Trichophyton mentagrophytes. (BOND, 2010).

Como as dermatofitoses são infecções contagiosas com elevada prevalência na América Latina (BETANCOURT et al., 2009) e caracterizadas como

IPrograma de Pós-graduação em Ciências Veterinárias, Universidade Federal de Mato Grosso (UFMT), 78060-900, Cuiabá, MT, Brasil. E-mail: rnvet@uol.com.br. *Autor para correspondência.

"Curso de Medicina Veterinária, UFMT, Cuiabá, MT, Brasil.

IIIDepartamento de Clínica Médica Veterinária, UFMT, Cuiabá, MT, Brasil. 
zoonose, não é raro observar animais, mas também o homem atingido por essa enfermidade (PINHEIRO et al., 1997). No homem, dados epidemiológicos indicam que essas micoses estão entre as zoonoses mais comuns do mundo, sendo considerado o terceiro distúrbio de pele mais comum em crianças menores de 12 anos e o segundo da população adulta (PINHEIRO et al., 1997) com a incidência e prevalência variando em função do clima e dos reservatórios naturais (MACIEL \& VIANA, 2005).

A enfermidade parece ser mais comum em climas tropicais e temperados, particularmente, em países com áreas de condições climáticas quentes e úmidas (CAVALCANTI et al., 2003), com maior incidência nos meses de outono e inverno (MACIEL \& VIANA, 2005). Em São Paulo, evidenciou-se que as dermatofitoses se situam em terceiro lugar entre as enfermidades cutâneas, sendo precedidas apenas pelas dermatites alérgicas e parasitárias (LARSSON et al., 1997). Em Saúde Pública, aproximadamente $15 \%$ de todos os quadros clínicos de dermatofitoses em humanos são de origem zoonótica (LARSSON et al., 1997).

As dermatofitoses apresentam como sinais clínicos mais comuns, alopecia, eritema, crostas e escamas (BOND, 2010). Em gatos, os principais carreadores (MENDOZA et al., 2009), os pêlos ficam tonsurados (CAVALCANTI et al., 2003). A enfermidade varia desde infecções agudas autolimitantes, geralmente sem prurido, podendo haver remissão espontânea, até quadros crônicos que persistem por meses ou anos (MACIEL \& VIANA, 2005).

Pentes, escovas, cortadores, camas, caixas de transporte e todo o material associado à tosa, manutenção e movimentação do animal em casa são fontes potenciais de infecção e reinfecção (MACIEL \& VIANA, 2005). Infecções por Trichophyton spp., geralmente, são adquiridas direta ou indiretamente por exposição a hospedeiros reservatórios típicos, os quais podem ser determinados por identificação específica da espécie ou subespécie fúngica. Por exemplo, a maioria das infecções por $\boldsymbol{T}$. mentagrophytes está associada à exposição a roedores ou ao seu ambiente imediato (BOND, 2010).

As dermatofitoses podem ocorrer em cães jovens que vivem em coletividade ou em cães com idade avançada que apresentam imunodepressão, os quais podem apresentar alopecia progressiva, localizada ou generalizada (KEENAM, 2007). Alguns estudos evidenciaram um maior acometimento de machos da espécie felina (CAVALCANTI et al., 2003), já outros referem inexistir predisposição sexual (BETANCOURT et al., 2009). Em relação às raças, cães Yorkshire Terrier e gatos da raça Persa parecem ter uma maior prevalência (SPARKES et al., 1993; BRILHANTE et al., 2003; CAFARCHIA et al., 2004). As razões para a predisposição de tais raças ainda não são bem claras. Possivelmente, os pêlos alongados facilitam as condições ótimas de temperatura e umidade para que as estruturas fúngicas fiquem protegidas contra a dissecação, favorecendo assim a sua propagação (SPARKES et al., 1993).

A alta ocorrência de dermatofitoses na causística local, a possibilidade de variação na incidência e prevalência de uma região geográfica para outra, bem como a importância da doença em questão motivaram a realização deste trabalho. Os objetivos deste estudo foram caracterizar a população canina e felina com dermatofitose atendida no Hospital Veterinário da Universidade Federal de Mato Grosso HOVET/UFMT.

\section{MATERIAL E MÉTODOS}

Foi realizado estudo dos casos atendidos no Setor de Clínica Médica do HOVET/UFMT, na Cidade de Cuiabá, Mato Grosso, no período de janeiro de 2006 a dezembro de 2008, através da análise retrospectiva dos prontuários. Com isso, buscando-se a obtenção de dados como: espécie; definição racial e raça; idade (>1 ano, 1 a 3 anos, >3 anos); sexo; relato de transmissão interespécie; sazonalidade (período chuvoso: novembro a maio, período de seca: junho a outubro); etiologia; configuração e topografia lesional e presença de prurido.

Fragmentos de pêlos e escamas da pele, retirados com auxílio de pinça ou raspagem cutâneo superficial, foram semeados em ágar Sabouraud com cloranfenicol no Laboratório de Microbiologia do HOVET/UFMT, sendo a caracterização do gênero e espécie de dermatófitos realizada segundo QUINN et al. (1994) .

As análises estatísticas foram realizadas pelo este do Qui-quadrado de Pearson, com nível de significância de 5\% (SOKAL \& ROOHLF, 1995).

\section{RESULTADOS}

No referido período, foram atendidos 3906 animais, dentre estes $451(11,5 \%)$ apresentaram dermatopatia. As dermatofitoses foram diagnosticadas em $279(61,9 \%)$ dos casos de dermatopatia, sendo 270 $(96,7 \%)$ na espécie canina e nove $(3,2 \%)$ em felinos, não havendo diferença estatística entre as espécies $(\mathrm{P}=0,064)$. A dermatopatia em questão totalizou 7,1\% (279/3906) do número de casos atendidos nesses três anos. 
Em relação aos cães, constatou-se que 58 $(21,5 \%)$ não apresentavam precisa definição racial e $212(78,5 \%)$ eram cães de raça definida, não ocorrendo diferença estatística entre esses dois grupos $(\mathrm{P}=0,79)$. A totalidade dos 270 cães encontra-se distribuída, por raça, a seguir: 46 da American Pit Bull Terrier; 20 Dachshund; 19 Pinscher; 19 Poodle; 13 Cocker S. Inglês; 12 Labrador; 10 Yorkshire; 9 Lhasa Apso; 9 Boxer; 8 Shih tzu; 6 Rottweiller; 5 Fila Brasileiro; 5 Fox Paulistinha; 5 Pastor Alemão; 4 Dálmata; 4 Maltês; 3 Pointer Inglês; 3 Bull Terrier; 2 Pastor Belga; 2 Beagle e oito exemplares unitários representando raças distintas (Akita; Australian Cattle Dog; Chow-chow; Dobermann; Dog Alemão; Schnauzer Miniatura; Shar Pei e Weimaraner). Relativamente aos gatos, constatou-se que $3(33,3 \%)$ dos animais não tinham definição racial e $6(66,7 \%)$ eram de raça definida, sendo quatro $(66,7 \%)$ da raça Persa e dois $(33,3 \%)$ da raça Siamesa.

A faixa etária dos cães variou entre um a 180 meses, tendo como idade média 40,8 meses (Moda = 24). Nos felinos, a variação de idade foi de 5 a 156 meses, sendo a média etária de 34,4 meses (Moda = 12). Observou-se que, dos 270 cães, 70 (25,9\%) apresentavam menos de um ano de idade no momento do primeiro atendimento e que, dos 9 gatos, apenas 1 $(11,1 \%)$ tinha menos de 12 meses, não ocorrendo significância estatística na faixa etária de cães $(\mathrm{P}=0,61)$ e nem na dos gatos $(\mathrm{P}=0,45)$.

Com relação ao sexo, do total de animais com dermatofitose, 123 eram machos e 147 eram fêmeas. Já, com relação aos felinos, quatro eram machos e cinco eram fêmeas. Assim, na totalidade, o número de casos em fêmeas foi superior ao número de machos independente da espécie, entretanto, estatisticamente, não houve significância para cães $(\mathrm{P}=0,74)$ e nem para gatos $(\mathrm{P}=0,36)$.

Quanto à possível transmissão interespécie, observaram-se quatro proprietários com lesões sugestivas de dermatofitose, sendo que três mantinham contato com cão e um com gato, sendo recomendada a procura por um médico dermatologista.

Na distribuição sazonal, observou-se que 162 animais vieram para a consulta no "período das chuvas" e 117 no "período seco", não havendo diferença estatística $(\mathrm{P}=0,89)$.

$\mathrm{O}$ agente Microsporum canis foi identificado em 270 (96,78\%) dos animais, Trichophyton spp. em $3(1,1 \%)$ e em $6(2,1 \%)$ não foi possível estabelecer o diagnóstico definitivo.

As lesões comumente observadas foram: alopecia $(62,4 \%)$; crostas $(39,4 \%)$; caspas $(21,1 \%)$; eritema $(8,2 \%)$; máculas $(7,5 \%)$; pústulas $(7,5 \%)$; pápulas $(5,7 \%)$; escoriações $(2,5 \%)$; hiperqueratose $(2,5 \%)$; foliculite $(2,1 \%)$; úlceras $(1,1 \%)$ e colaretes epidérmicos $(1,1 \%)$. Nos cães, as lesões encontravamse distribuídas no tronco $(60,7 \%)$, na cabeça $(51,5 \%)$, nos membros (48,5\%), no abdômen $(26,6 \%)$, na região cervical $(18,9 \%)$, na região lombossacra $(18,1 \%)$ e na região coccígena $(13,3 \%)$. Nos felinos, as lesões apresentavam-se distribuídas no tronco (7), na cabeça (4), nos membros (2), na região abdominal (1), na região cervical (5) e na cauda (1).

A manifestação clínica de prurido foi constatada em 173 cães, sendo 71 de forma leve, 30 moderada e 72 intensa. Nos gatos, verificou-se que, em um animal, o prurido era leve, em três, moderado e em dois, intenso.

\section{DISCUSSÃO E CONCLUSÃO}

No presente estudo, a ocorrência de 7,14\% em relação ao total de casos novos atendidos, no período de 36 meses, nas espécies canina e felina corrobora o achado de autores europeus e da América do Norte. Eles relatam que esta infecção varia no intervalo de 7 a $40 \%$ em cães e de 9 a 54,8\% em gatos. Além disso, os resultados desta pesquisa não estão longe dos achados sugestivos de dermatofitoses na cidade de Fortaleza-CE, os quais demonstraram 14,3\% dos caninos e 36,8\% dos felinos acometidos por diferentes espécies de dermatófitos (BRILHANTE, 2005). Similarmente, ainda no Nordeste, foram identificados dermatófitos em 13,8\% dos cães e gatos amostrados (CAVALCANTI et al., 2003).

No que se refere à definição racial e à raça, constatou-se que, com relação aos animais da espécie canina, maior prevalência entre os com definição racial, sem, no entanto, ocorrer significância estatística. Destes, o maior número coube aos da raça American Pit Bull Terrier, com 46 (21,7\%) exemplares. Esses dados corroboram outros estudos no que se refere à definição racial (SPARKES et al., 1993), porém se contrapõem com a maioria que coloca a raça Yorkshire Terrier como a mais predisposta a desenvolver infecções fúngicas (SPARKES et al., 1993; BALDA et al., 2004; MACIEL \& VIANA, 2005; CAVALCANTE, 2006). As razões para a predisposição de tais raças ainda não estão bem claras. Para alguns autores, possivelmente, os pêlos alongados contribuem para que as estruturas fiquem protegidas da dissecação, favorecendo a sua propagação, devido às ótimas condições de temperatura e umidade, favorecendo a sua propagação (SPARKES et al., 1993). Para outros, isso se deve às diferenças nas defesas cutâneas, não específicas, como secreção sebácea/ sudorípara e não devido ao seu pêlo longo 
(CAFARCHIA et al., 2004). De forma similar, não se encontrou distinção entre os felinos com ou sem definição racial, mas, dentre os gatos que a apresentavam, notou-se que a maioria era Persa. Esse fato deve ser analisado com cautela pelo tamanho amostral, podendo esta predisposição racial estar relacionada a características hereditárias e/ou devido ao fato de os pêlos longos alojarem conídios com maior facilidade (SPARKES et al., 1993; MACIEL \& VIANA, 2005). Os dados contradizem um estudo de dermatofitose humana em que se conclui que pêlos mais curtos, em casos de Tinea capitis, beneficiaram a implantação e instalação de artroconídios (BRILHANTE, 2005). Ainda, para os gatos da raça Persa, existe a teoria de que a predisposição estaria apoiada no fato de que animais desta raça são mais, rotineiramente, suscetíveis de serem mantidos juntos, em grandes grupos, facilitando a disseminação do microorganismo(CAFARCHIA et al., 2004).

Quanto à faixa etária dos animais, esta variou de um mês a 15 anos nos cães e de 5 a 13 anos nos gatos. Embora tenha sido alta a presença de dermatofitose nos animais com até 12 meses, 25,93\% nos cães e $11,11 \%$ nos gatos, uma maior percentagem foi apontada nos animais com idade entre 1-3 anos, $33,33 \%$ nos cães e $55,56 \%$ nos gatos, diferentemente dos outros trabalhos revisados que colocam os animais situados no primeiro ano de vida como de maior predisposição para a infecção (BRILHANTE et al., 2003; BALDA et al., 2004; CAFARCHIA et al., 2004).

Com relação ao sexo dos cães e gatos, não se observou diferença, concordando com outros estudos (SPARKES et al., 1993;LARSSON et al., 1997; BALDA et al., 2004). Entretanto, CAFARCHIA et al.(2004) encontraram uma maior proporção de machos, relacionando esta maior suscetibilidade à característica de produzir maior secreção sebácea.

No que se refere à possível transmissão interespécie, observou-se o relato de quatro proprietários com lesões sugestivas de dermatofitose durante a consulta dos animais, três $(1,1 \%)$ em contato com cães positivos e outra pessoa $(0,04 \%)$ com contato com um gato nas mesmas condições. NOBRE et al. (2001), estudando a importância do felino na epidemiologia da dermatofitose, comprovou que um animal desta espécie transmitiu M. canis para os cinco membros da família, já outro estudo identificou que em 111 casos de dermatofitose humana, 23 foram por contato com animais de companhia (BASSANESI et al., 1993). Esse tipo de transmissão, cão/gato para o homem se confirma também em outros estudos (CAFARCHIA et al., 2006).

No que concerne à sazonalidade, observase que ocorreu um maior número de consultas com animais positivos $(58,1 \%)$ para dermatofitose no período de março, abril e maio, sem, no entanto, ocorrer diferença estatisticamente significativa, apesar de coincidir, grande parte deste período, com a estação chuvosa em Mato Grosso. Assim, houve a confirmação do estudo executado na região nordeste, que encontrou a maior porcentagem de prevalência nos mesmos meses (BRILHANTE, 2005). Também ratifica a pesquisa de influência sazonal em São Paulo, que identificou os maiores índices de dermatofitose nas estações de primavera, verão e outono (LARSSON et al., 1997), confirmando maior incidência de dermatofitose em período quente e úmido (SCOTT et al., 1996).

O agente $M$. canis foi a principal espécie isolada, totalizando $270(96,77 \%)$ animais atingidos, coincidindo com outras pesquisas (BRILHANTE, 2005; PRADO et al., 2008), seguido do Trichophyton sp. com $6(1,07 \%)$ animais, concordando com outros autores (LARSSON et al., 1997). Entretanto, em outros estudos, o segundo dermatófito mais encontrado foi o $\boldsymbol{M}$. gypseum (COSTA et al., 1994). Em um estudo realizado na região rural de Pernambuco, os dermatófitos acometeram $13,16 \%$ dos cães e $27,27 \%$ dos gatos, sendo também o gênero Microsporum o mais isolado. Esses dados coincidem com diagnóstico humano, no qual estudos apontam o $\boldsymbol{M}$. canis como o zoofílico mais isolado em Tinea capitis na cidade de São Paulo e Fortaleza. (BRILHANTE, 2005).

Com relação às lesões, pôde ser observada uma vasta diversidade nos animais que apresentavam dermatofitose. A alopecia foi o achado clínico de maior frequência $(62,4 \%)$, seguido por crostas $(39,4 \%)$ e, em menor concentração, o colarete epidérmico com porcentagem de $1,1 \%$, concordando com estudos do HOVET/USP (BALDA et al., 2004).

Referindo-se à topografia das lesões, notase que os achados desta pesquisa diferem do estudo realizado por BALDA et al. (2004), que relatam a região cervical como a de maior concentração de lesões (BALDA et al., 2004), além de estudos com felinos, nos quais relatam casos apresentando maior concentração de lesões na região dorso-lombar e base da cauda, como nas pesquisas de TOSTES \& GIUFFRIDA (2003).

A maioria dos animais atendidos apresentava prurido $(64,2 \%)$, apesar de este não ser um fator determinante no diagnóstico de dermatofitose, conforme estudo mostrando que apenas $23,3 \%$ dos cães e gatos com quadro clínico de prurido e sintomas de dermatofitoses produziram culturas positivas (CAFARCHIA et al., 2004). Isso enfatiza as dificuldades de se fazer um diagnóstico de dermatofitose baseado 
em sinais clínicos e lesões cutâneas, realçando a necessidade de confirmação laboratorial através da cultura fúngica, antes de se instituir um tratamento (CAFARCHIA et al., 2004).

A ocorrência de dermatofitose foi de 7,14\% em cães e gatos, com maior frequência nas fêmeas, em cães adultos jovens, com raça definida. Preponderantemente, em ambas as espécies, as lesões alopécicas e crostosas localizavam-se na região torácica e estavam mais frequentemente associadas à presença de M.canis.

\section{REFERÊNCIAS}

BALDA, A.C. et al. Estudo retrospectivo de casuística das dermatofitoses em cães e gatos atendidos no Serviço de Dermatologia da Faculdade de Medicina Veterinária e Zootecnia da Universidade de São Paulo. Acta Scientiae Veterinariae, v.32, n.2, p.133-140, 2004. Disponivel em: <http:// www.ufrgs.br/actavet/32-2/artigo590.pdf>. Acesso em: $20 \mathrm{dez}$. 2009.

BASSANESI, M.C. et al. Fonte de infecção na dermatofitose por Microsporum canis. Anais Brasileiros de Dermatologia, v.68, n.1, p.11-13, 1993. Disponível em: <http:// www.anaisdedermatologia.org.br/public/figuras.aspx ?id=1034>. Acesso em: 25 jun. 2010.

BETANCOURT, O. et al. Microsporum canis em gatos dermatologicamente sanos en Temuco, Chile. Iberoamericana de Micologia, v.26, n.3, p.206-210, 2009. Disponivel em $<\mathrm{h} \mathrm{t} \mathrm{t} \mathrm{p} \mathrm{:} \mathrm{/} \mathrm{/} \mathrm{w} \mathrm{w} \mathrm{w} \mathrm{.} \mathrm{s} \mathrm{c} \mathrm{i} \mathrm{e} \mathrm{n} \mathrm{c} \mathrm{e} \mathrm{d} \mathrm{i} \mathrm{r} \mathrm{e} \mathrm{c} \mathrm{t} \mathrm{.} \mathrm{c} \mathrm{o} \mathrm{m} \mathrm{/}$ science?_ob=ArticleURL\&_udi=B 985 Y-4WTYY6T\&_user $=10 \&$ \&coverDate $=09 \% 2 \mathrm{~F} 30 \% 2 \mathrm{~F} 2009 \&$ \&doc $=1 \&$ fm $\mathrm{t}=\mathrm{high} \&$ orig $=$ search\&_sort $=\mathrm{d} \& \_$docanchor $=\&$ view $=\mathrm{c} \&$ _searc hStrId=1388551826\&_rerunOrigin=google \&_acct $=$ C000050221\&_ version $=1 \& \_$urlVersion $=0 \& \_$userid $=10 \& \mathrm{md} 5=3$ eeab7fd635727c92baa0f8 26d388f4b. Acesso em: 16 jun. 2010. doi:10.1016/ j.riam.2009.03.002.

BOND, R. Superficial veterinary mycoses. Clinics in Dermatology, v.28, p.226-236, 2010. Disponivel em: <http:/ / w w w. s c i e $n$ c e d i r e c t c c o m / science?_ob=ArticleURL\&_udi=B6T5G-4YP86P5N\&_user=10\&_coverDate $=03 \% 2 \mathrm{~F} 04 \% 2 \mathrm{~F} 2010$ \&_rdoc $=1 \&$ fmt $=$ high $\&_{-}$orig=searchhttp://www.sciencedirect.com/ science?_ob=ArticleURL\&_udi=B6T5G-4YP86P5N\&_user $=10 \&$ _coverDate $=03 \% 2 \mathrm{~F} 04 \% 2 \mathrm{~F} 2010 \&$ \&doc $=1 \&$ fm $\mathrm{t}=$ high \&_orig=search\&_sort=d\&_docanchor $=\& \mathrm{view}=\mathrm{c} \& \_s e a r c h S t r I d$ $=1388619020 \&$ \&rerunOrigin $=$ google $\& \_$acct $=\mathrm{C} 000050221 \&$ _version $=1 \&$ \&urlVersion $=0 \& \_u s e r i d=10 \& \mathrm{md} 5=9 \mathrm{c} 8 \mathrm{e} 2 \mathrm{ac} 9167 \mathrm{eb} 0$ $63 \mathrm{f} 13537$ cbee9e $3839 \& \_$sort $=\mathrm{d} \&$ docanchor $=\& \mathrm{view}=\mathrm{c} \&$ _sear chStrId $=1388619020 \&$ _rerunOrigin $=$ google $\& \_a c c t=C 0000502$ $21 \&$ version $=1 \&$ urlVersion $=0 \& \_u s e r i d=10 \& m d 5=9 \mathrm{c} 8 \mathrm{e} 2 \mathrm{ac} 9167 \mathrm{eb} 063 \mathrm{f} 135$ 37cbee9e3839>. Acesso em: 10 jun. 2010. doi:10.1016/ j.clindermatol.2009.12.012.

BRILHANTE, R.et al. High rate of Microsporum canis feline and canine dermatophytoses in Northeast Brazil: epidemiological and diagnostic features. Mycopathologia, v.156, n.4, p.303308, 2003. Disponivel em: <https://springerlink.metapress.com/ content/m054222j50235403/resource-secured/ ?target=fulltext.pdf\&sid=2c1a34f4uqljwr45rnwbcxin\&sh=www.spr
ingerlink.comh\&_sort=d\&_docanchor=\&view=c\&_searchStrId $=138857$ $2822 \&$ rerunOrigin $=$ google \&_acct $=\mathrm{C} 000050221 \&$ \& version $=1 \&$ _urlVersion=0\&_userid=10\&md5=33b8fb5730182351407910da7c6a992>. Acesso em: 19 jun. 2010. doi:10.1/j.clin dermatol.2009.12.012

BRILHANTE, R.S.N. Caracterização fenotípica e genotípica de Microsporum canis oriundos de cães e gatos como um possível clone fúngico. 2005. 82f. (Doutorado em Reprodução e Sanidade Animal) - Faculdade de Veterinária, Universidade Estadual do Ceará, CE.

CAFARCHIA, C.et al. Isolation of Microsporum canis from the hair coat of pet dogs and cats belonging to owners diagnosed with M. canis tinea corporis. Veterinary Dermatology, v.17, n.5, p.327331, 2006. Disponivel em: <http://www3.interscience.wiley.com/ journal $/ 118573623 /$ abstract?CRETRY $=1 \&$ SRETRY $=0>$. doi10.1111/j.1365-3164.2006.00533. Acesso em: 19 jun. 2010.

The epidemiology of canine and feline dermatophytoses in southern Italy. Mycoses, v.47, n.11-12, p.508-513, 2004. Disponivel em: <http://www3.interscience.wiley.com/journal/ 118789163/abstract>. Acesso em: 16 jun. 2010. doi: 10.1111/ j.1439-0507.2004.01055.

CAVALCANTE, C.S.D.P. Caracterização das dermatofitoses canina e felina e manutenção das cepas dermatofíticas in vitro. 2006. 90f. (Mestrado em Reprodução e Sanidade Animal) - Programa de Pós-graduação Em Ciências Veterinárias, Universidade Estadual do Ceará, CE.

CAVALCANTI, M.D.P. et al. Frequência de dermatófitos e fungos saprófitas em caninos e felinos com sintomatologia sugestiva de dermatopatia micótica atendidos no Hospital Veterinário da UFRPE. Revista clínica Veterinária, v.56, p.24-28, 2003.

COSTA, E. et al. Interspecific outbreaks of dermatomycoses +caused by Microsporum canis and Microsporum gypseum. Revista Saude Publica, v.28, n.5, p.337-340, 1994. Disponível em: <http://www.ncbi.nlm.nih.gov/pubmed/ 7660034>. Acesso em: 17 jun. 2010.

KEENAM, L.D. Micoses superficiais - Dermatofitoses. Nosso Clínico, n.57, p.6-14, 2007.

LARSSON, C.E. et al. Dermatofitoses de cães e gatos em São Paulo: estudo da possível influência sazonal. Sociedade Brasileira de Dermatologia, v.72, n.2, p.139-142, 1997. Disponível em: <http://www.anaisdedermatologia.org.br/public/ figuras.aspx ?id=644>. Acesso em: 23 jun. 2010.

MACIEL, A.S. ,VIANA, J.A. Dermatofitose em cães e gatos primeira parte. Revista Clínica Veterinária, v.56, p.48-56, 2005.

MENDOZA, M.H. et al. A zoonotic ringworm oubreak caused by a dysgonic strain of Microsporum canis from stray cats. Revista Iberoamericana de Micologia, v.27, n.2, p.62-65, 2009. Disponivel em: <http://www.reviberoammicol.com/201027/index.shtml>. Acesso em: 16 jun. 2010. doi:10.1016/ j.riam.2009.12.007.

NOBRE, M.D.O.et al. Importância do felino doméstico na epidemiologia da dermatofitose por Microsporum canis. Revista da Faculdade de Zootecnia, Veterinária e Agronomia, v.7/8, n.1, p.84-91, 2001. Disponivel em:<http:/ 
/revistaseletronicas.pucrs.br/ojs/index.php/fzva/article/viewFile/ 2060/1554>. Acesso em: 23 jun. 2010.

PINHEIRO, A.D.Q. et al. Dermatofitose no meio urbano e a coexistência do homem com cães e gatos. Revista da Sociedade Brasileira de Medicina Tropical, v.30, n.4, p.287-294, 1997. Disponivel em: <http://www.scielo.br/scielo.php?pid=S0037$86821997000400003 \&$ script=sci_arttext $>$. Acesso em: 01 jul. 2010. doi: $10.1590 /$ S0037-86821997000400003.

PRADO, M. et al. Frequency of yeasts and dermatophytes from healthy and diseased dogs. Journal of Veterinary Diagnostic Investigation, v.20, n.2, p.197-202, 2008. Disponível em: <http://jvdi.org/cgi/content/full/20/2/197>. Acesso em: 01 jul. 2010.

QUINN, P.J. et al. Clinical Veterinary mocrobiology. London: Wolfl, 1994. 648p.
SCOTT, D.W. et al. Muller \& Kirk - Dermatologia de pequenos animais. 5.ed. Rio de janeiro: Interlivros, 1996. 1130p.

SOKAL, R.R.; ROHLF, F.J. Biometry. 3.ed. New York: W.H. Freeman, 1995. 887p.

SPARKES, A.et al. Epidemiological and diagnostic features of canine and feline dermatophytosis in the United Kingdom from 1956 to 1991. Veterinary Record, v.133, n.3, p.57-61, 1993. Disponivel em: <http://veterinaryrecord.bvapublications.com/ cgi/content/abstract/133/3/57>. Acesso em: 20 jun. 2010.

TOSTES, R.A.; GIUFFRIDA, R. Pseudomicetoma dermatofítico em felinos. Revista Ciência Rural, v.33, n.2, p.363-365, 2003. Disponivel em: <http://www.scielo.br/ scielo.php? script $=$ sci_arttext \& pid $=$ S $0103-$ 84782003000200028>. Acesso em: 05 jul. 2009. doi:10.1590/S0103-84782003000200028. 